\title{
Detection of hepatocellular carcinoma by tissue resonance interaction method (TRIM)
}

\author{
Grzegorz Boryczka ${ }^{1}$, Marek Hartleb ${ }^{1}$, Małgorzata Janik ${ }^{2}$ \\ ${ }^{1}$ Department of Gastroenterology and Hepatology, School of Medicine, Medical University of Silesia, Katowice, Poland \\ ${ }^{2}$ Department of Biomedical Computer Systems, Institute of Informatics in Sosnowiec, University of Silesia, Sosnowiec, Poland
}

Gastroenterology Rev 2018; 13 (1): 40-46

DOI: https://doi.org/10.5114/pg.2018.74561

Key words: diagnosis, hepatocellular carcinoma, tissue resonance interaction.

Address for correspondece: Grzegorz Boryczka MD, PhD, Department of Gastroenterology and Hepatology, Medical University of Silesia, 14 Medyków St, 40-752 Katowice, Poland, phone: +48 3278944 01, fax: +48 32789 44 02, e-mail: grzegorzboryczka@op.pl

\begin{abstract}
Introduction: Diagnosis of hepatocellular carcinoma (HCC) is considerably delayed, being frequently done in the non-curative stage of disease. The reason for delayed diagnosis is indolent course in early stages and/or unspecific symptoms indistinguishable from underlying cirrhosis. Hitherto methods used for screening of HCC have important limitations. TRIMprob is a non-invasive method, which showed utility in detection of cancers located in prostate, breast, or urinary bladder.

Aim: To determine the diagnostic accuracy of TRIMprob in detecting HCC in cirrhotic liver.

Material and methods: Forty-five patients were prospectively enrolled according to final clinical diagnosis into a group of cirrhosis and HCC or a group of cirrhosis without HCC. A control group consisted of 33 healthy subjects. Hepatocellular carcinoma was diagnosed by computed tomography (CT) or magnetic resonance (MR) and guided biopsy. The TRIMprob examination was performed in each patient. Three wave frequencies were used: 465, 930, and $1395 \mathrm{MHz}$.

Results: In patients with HCC the intensity of return signal using wave a frequency of $465 \mathrm{MHz}$ was significantly reduced in patients with HCC in comparison to healthy subjects $(p<0.0005)$, but not to cirrhotic patients without HCC. Moreover, cirrhosis was associated with significantly decreased TRIMprob signal in comparison to healthy liver $(p<0.002)$. In ROC analysis an optimal cut-off value for detection of HCC was 106 units, which yielded 80\% sensitivity.

Conclusions: TRIMprob identifies HCC with good sensitivity; however, the accuracy of this method to identify HCC in screening circumstances may be hindered by attenuation of the resonance interaction signal by cirrhosis itself.
\end{abstract}

\section{Introduction}

Hepatocellular carcinoma (HCC) is diagnosed yearly in more than 500,000 people worldwide [1]. It is the fifth most common cancer in men and the seventh in women, and it among the most aggressive of human tumours [2]. In Europe the incidence of HCC ranges from 2.9 to 5.2 cases per 100,000 [3]. Generally, this tumour shows a tendency towards increasing prevalence in developed countries, but its diagnosis is considerably delayed, being frequently done in the non-curative stage of disease. The reason for delayed diagnosis is indolent course in early stages and/or unspecific symptoms indistinguishable from underlying cirrhosis. The major risk factor for the development of HCC is liver cirrhosis of viral, alcoholic, or metabolic aetiology $[2,4]$. The European Association for the Study of the Liver (EASL) and European Organisation for Research and Treatment of
Cancer (EORTC) established criteria for diagnosis of HCC that incorporate both invasive and noninvasive measures [2]. Generally, the diagnosis of HCC is based on contrast imaging methods, and liver biopsy is reserved for diagnostically inconclusive cases.

Improving survival in HCC depends on access to sensitive, non-invasive, and cost-effective methods suitable for wide-ranged screening in high-risk populations. Tissue resonance interaction is a new method that analyses electromagnetic anisotropy in organic tissues. TRIMprob generates an alternating electromagnetic field that interacts with charged particles in a target tissue, leading to a secondary radiation being a source return signal that varies for normal and neoplastic tissue [5]. Diagnosis of cancer by TRIMprob is mostly based on detection of the microtubules, which are cellular structures participating in mitotic division, more precisely in separation of chromatids [6]. The presence of 
microtubules is characteristic for rapidly dividing cells. Using a wave frequency equal to half of the microtubule length was found to optimally detect cancerous growth. Several pilot clinical studies have shown that TRIMprob scanning is a valuable tool in diagnosing cancers developing in prostate [7-11], breast [12], stomach [13], thyroid gland [14], rectum [15], colon [16], or urinary bladder [17, 18].

\section{Aim}

The aim of this study was to determine the diagnostic accuracy of TRIMprob in detecting HCC.

\section{Material and methods}

This study was conducted in a tertiary referral centre. Forty-five patients aged 22 to 85 years $(56.3 \pm 15.0$ years) hospitalised in our department were prospectively enrolled according to final clinical diagnosis either to the group of cirrhosis and HCC $(n=10)$ or to cirrhosis without HCC $(n=35)$. General demographic characteristics of subjects are shown in Table I. The control group consisted of 33 healthy persons without history of liver or neoplastic disease, who did not show abnormalities at abdominal ultrasound and had normal transaminases activity.

The eligibility criteria for the study were: age $>18$ years (for all subjects) and presence of compensated or decompensated cirrhosis. The exclusion criteria were: body mass index (BMI) $\geq 40 \mathrm{~kg} / \mathrm{m}^{2}$, massive ascites, advanced encephalopathy, severe heart or lung failure, pacemaker implantation, the inability to maintain upright position, hepatic tumour other than HCC, and pregnancy.

The diagnosis of liver cirrhosis was based on a combination of clinical, laboratory, radiological, endoscopic, and histopathological criteria. Arterial hypervascularity and venous/late phase washout were key criteria for diagnosis of HCC at multiphase computed tomography (CT) or dynamic contrast enhanced magnetic resonance (MR). Ultrasound-guided tumour biopsy was essential for the diagnosis of HCC in only 4 patients.

Examination by TRIMprob was performed by a single operator who, after short-term training, used in each patient the constant transabdominal "windows" specified in previous studies $[13,16]$. During examination the patients were dressed in pyjamas or T-shirts, in an upright position, in front of the base of the device. The operator was standing on the right side of the patient with the probe in his right hand and touched the surface of the abdomen covered by clothing (Figure 1). The duration of examination was less than 5 min.

The probe emitted electromagnetic radiation of strength similar to that experienced during the use of a cordless telephone, of three frequencies: $465 \mathrm{MHz}$, $930 \mathrm{MHz}$, and $1395 \mathrm{MHz}$. A spectrum analyser, fed by a receiving antenna, measured signal intensities that were graphically displayed on a computer screen, and the result was expressed in arbitrary units ranging between 0 and 255.

The study conformed to the ethical guidelines of the 1975 Declaration of Helsinki ( $6^{\text {th }}$ revision, 2008). All patients received detailed information about the pur-

Table I. Demographic and laboratory data of patients with liver cirrhosis with and without HCC

\begin{tabular}{|c|c|c|c|}
\hline Variable & $\begin{array}{l}\text { Cirrhosis without HCC } \\
\qquad(n=35)\end{array}$ & $\begin{array}{l}\text { Cirrhosis with HCC } \\
\qquad(n=10)\end{array}$ & $P$-value \\
\hline Age, mean \pm SD [years] & $53.8 \pm 14.6$ & $64.9 \pm 13.9$ & 0.0757 \\
\hline Sex (male/female), $n$ & $20 / 15$ & $5 / 5$ & 0.9680 \\
\hline $\mathrm{BMI}$, mean $\pm \mathrm{SD}\left[\mathrm{kg} / \mathrm{m}^{2}\right]$ & $27.0 \pm 4.6$ & $26.6 \pm 5.2$ & 0.9424 \\
\hline Haemoglobin, mean $[\mathrm{g} / \mathrm{dl}]$ & 13.2 & 11.9 & 0.4798 \\
\hline Platelets, mean $\left[\times 10^{3} / \mathrm{mm}^{3}\right]$ & 161 & 140 & 0.3324 \\
\hline Bilirubin, mean [mg/dl] & 1.37 & 1.24 & 0.5480 \\
\hline Albumin, mean $[\mathrm{g} / \mathrm{dl}]$ & 2.90 & 3.80 & 0.5330 \\
\hline Alanine aminotransferase, mean [U/l] & 42.5 & 40.0 & 0.8164 \\
\hline Aspartate aminotransferase, mean [U/I] & 83.5 & 58.0 & 0.9022 \\
\hline INR, mean & 1.16 & 1.23 & 0.5199 \\
\hline$\alpha$-Fetoprotein, mean $[\mathrm{ng} / \mathrm{ml}]$ & 11.3 & 3.58 & 0.1201 \\
\hline
\end{tabular}

In 33 control subjects (17 females) the mean age was $35.0 \pm 11.3$ years, BM/ was $29.3 \pm 3.2 \mathrm{~kg} / \mathrm{m}^{2}$. INR - international normalised ratio. 


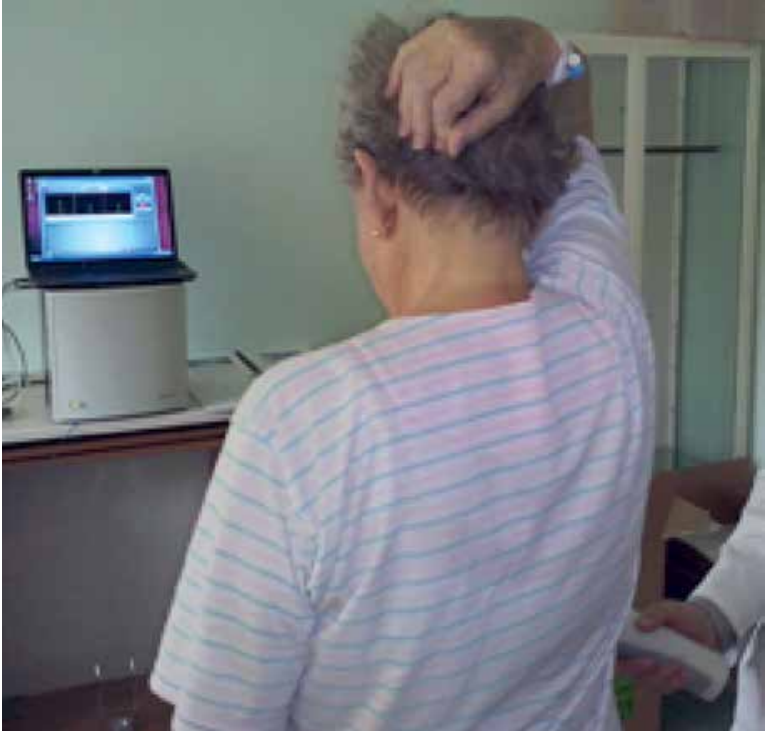

Figure 1 . The position of the patient during TRIMprob examination

pose and character of the study and gave consent to participate.

\section{Statistical analysis}

Statistical analysis was conducted using Statistica version 12 software (StatSoft; Krakow, Poland). The distribution of quantitative variables was tested using the Shapiro-Wilk test and evaluated graphically by means of histograms. For variables having normal distribution, the means and standard deviations were calculated; for variables deviating from normal distribution, the median values were chosen. One-way ANOVA (one-way analysis of variance) or unpaired t test was applied to check the differences between the means of the studied groups. In the case of variables that did not meet the required assumptions, the Kruskal-Wallis one-way ANOVA or U Mann-Whitney test was used. ROC (Receiver Operating Characteristic) curves were used to evaluate the diagnostic test and determine the cut-off point. To define the relationships between the variables, analyses of correlations and regressions were conducted. The $\chi^{2}$ test with Yates correction was used to evaluate the relationship between gender and belonging to a group. A $p$ level less than 0.05 was considered statistically significant.

\section{Results}

Alcoholic aetiology of cirrhosis was diagnosed in $11 \mathrm{pa}$ tients, chronic viral hepatitis B or C in 11 patients, autoimmune hepatitis in 12 patients, and metabolic (non-alcoholic steatohepatitis, Wilson disease, hereditary haemochromatosis) in 5 patients. No clear aeti- ology of cirrhosis could be determined in 6 patients. Mild or moderate ascites was found in 10 patients. The mean diameter of HCC measured on ultrasound was $42.6 \pm 13.2 \mathrm{~mm}$. Mean value of $\alpha$-fetoprotein (AFP) was $803 \pm 2434 \mathrm{ng} / \mathrm{ml}$ in cirrhotic patients with HCC and $7.6 \pm 9.1 \mathrm{ng} / \mathrm{ml}$ in those without HCC.

The analysis of tissue resonance interaction by TRIMprob for frequency waves of $465 \mathrm{MHz}, 930 \mathrm{MHz}$, and $1395 \mathrm{MHz}$ is shown in Figure 2. For the frequency of $465 \mathrm{MHz}$ the tissue resonance signals in patients with HCC, but also with cirrhosis without HCC, were significantly reduced in comparison to the individuals with healthy liver $(p=0.00025$ and $p=0.0015$, respectively). Tissue resonance signals in cirrhotic patients with and without HCC were not statistically different for any wave frequency. For the frequency of $1395 \mathrm{MHz}$ the TRIMprob signal was decreased in cirrhosis without $\mathrm{HCC}$ in relation to healthy liver $(p=0.00046)$. Significant correlations were found between TRIMprob signal intensity (at $1395 \mathrm{MHz}$ ) and serum levels of bilirubin $(r=-0.80007 ; p=0.0054)$ and albumin $(r=0.79235$; $p=0.0336$ ) (Figure 3). There were no significant correlations between signal intensity and such parameters as the tumour size, levels of transaminases, or platelet count.

In ROC analysis an optimal cut-off value for discrimination of HCC from healthy liver (at $465 \mathrm{MHz}$ ) was 106 units, which yielded $80 \%$ sensitivity and $94 \%$ specificity (area under ROC curve was 0.865) (Figure 4). In this analysis the discrimination of cirrhosis with HCC from cirrhosis alone provided the same cut-off value and sensitivity but lower specificity (66\%) with positive predictive value of $40 \%$ (area under ROC curve was 0.713). Table II summarises TRIMprob accuracy in discrimination between cirrhosis plus HCC and cirrhosis alone. When a two-step approach to diagnosis of HCC was tested, based on lower signal intensity than 106 units (465 MHz, first step) and higher signal intensity than 79 units (1395 MHz, second step), the diagnostic sensitivity was $90 \%$ and specificity was $77.1 \%$.

\section{Discussion}

Surveillance of cirrhotic patients for detection of early HCC is realised by 6-month interval abdominal ultrasound and serum level of AFP. The diagnostic sensitivity of ultrasound ranges from $58 \%$ to $89 \%$ [19-21]. Although the ultrasound is a readily available, non-invasive, and relatively cheap method, the detection of early HCC in a fibrotic background may be challenging because the coarse pattern of hepatic echogenicity may deteriorate identification of small tumours. Because of these limitations, the performance of ultrasound in detection of early HCC is dependent on the expertise of 
A

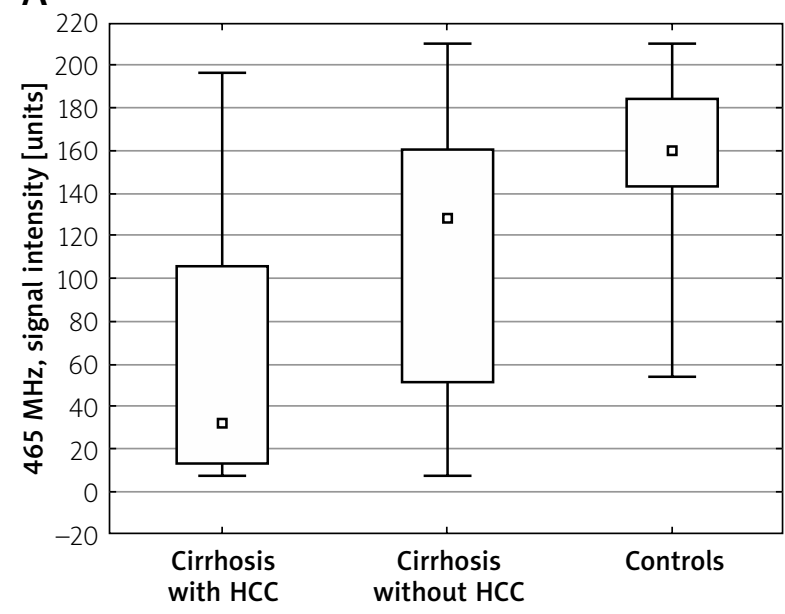

C

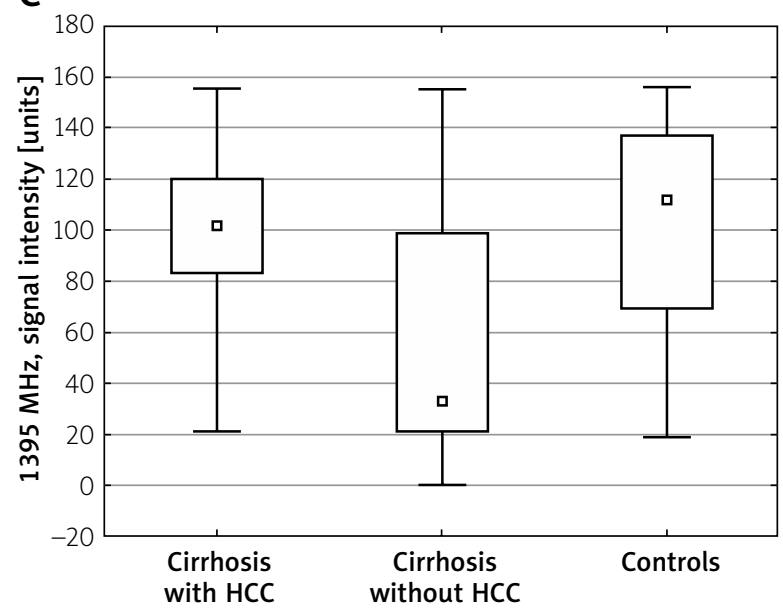

A

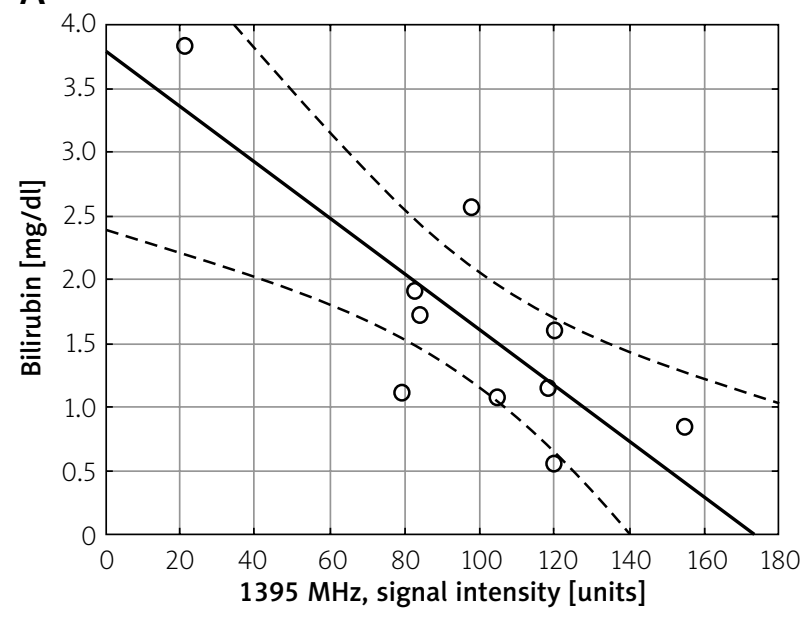

B

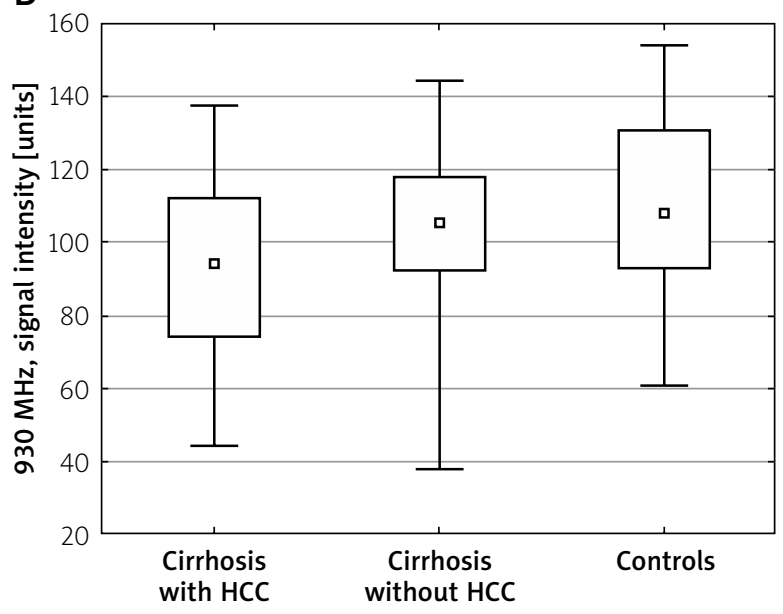

Figure 2. TRIMprob signal intensities registered in two groups of patients and healthy subjects at different frequency waves
B

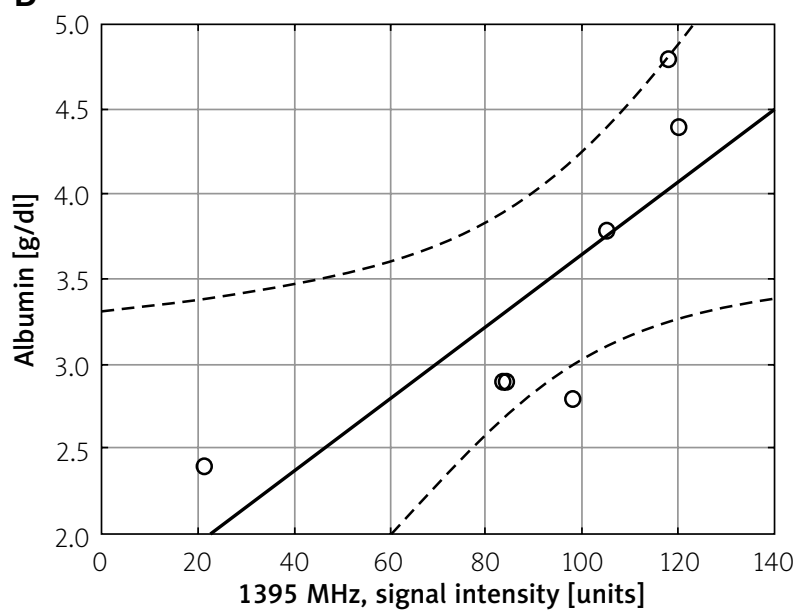

Figure 3. The correlations between TRIMprob signal intensity and laboratory indices of liver function (bilirubin, albumin) for $1395 \mathrm{MHz}$ wave frequency

the operator and the quality of the equipment [2]. AFP serum level exceeding $20 \mathrm{ng} / \mathrm{ml}$ shows average sensitivity of $60 \%$ in diagnosis of HCC [22]. Setting a cut-off value at $200 \mathrm{ng} / \mathrm{ml}$ is associated with perfect specificity but a significant decline of the sensitivity to $22 \%$ [23]. Moreover, fluctuating serum levels of AFP in patients 


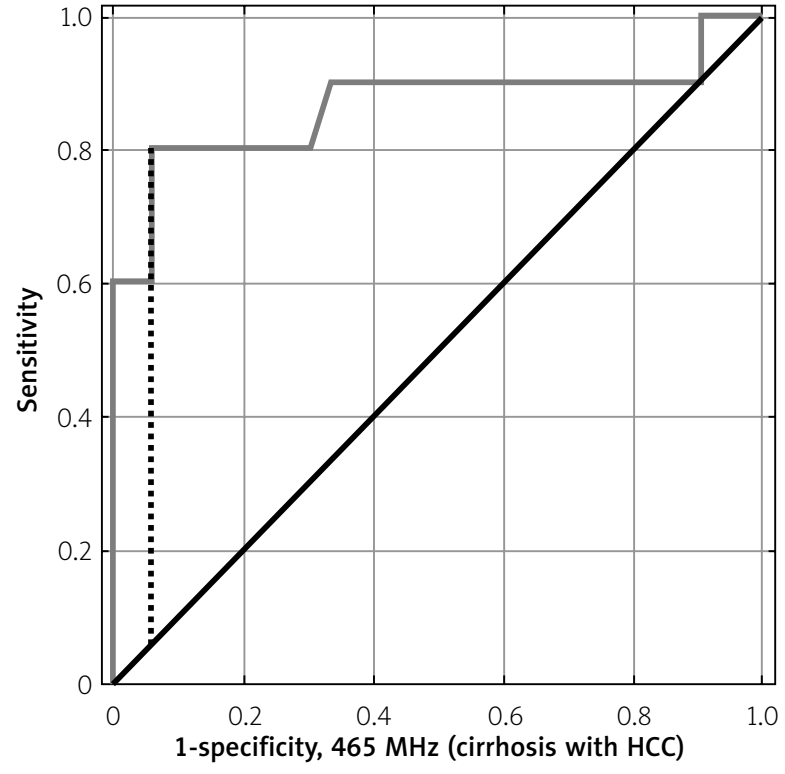

Figure 4. The ROC curves graph for accuracy of TRIMprob $(465 \mathrm{MHz})$ to identify hepatocellular carcinoma (HCC)

with cirrhosis may reflect bursts of viral replication or exacerbations of underlying liver disease [24]. For these reasons AFP is not recommended by EASL and EORTC for detection of early HCC [2]. Limitations of hitherto used methods to detect HCC arising in cirrhotic liver warrant the search for new non-invasive surveillance methods.

Vedruccio and Meessen put forward an idea of cancer detection based on non-linear resonance interactions with microtubules, representative for rapidly growing cells [5]. The electromagnetic waves generated by the TRIMprob stimulate minute electrical oscillations in biological tissues, which produce resonance effect of the magnitude dependent on the structural characteristics these tissues [7]. The receiver of the TRIMprob detects the signal originating from non-linear resonance interactions of intensity expressed in arbitrary units ranging between 0 and 255. TRIMprob is a simple, quick to perform (in this study examina- tion took less than $5 \mathrm{~min}$ ) test that does not require any prior preparation of the patient, yielding immediate and reproducible results $[5,6,11]$. Previous clinical studies have shown that TRIMprob scanning is useful in detecting cancers of different localisations. In a study by Bellorofonte et al. performed on 757 patients with prostate cancer TRIMprob showed good performance in detection of this neoplasm at a frequency of $465 \mathrm{MHz}$ [7]. In other validating studies the diagnostic sensitivity of TRIMprob was $80 \%$ and $86 \%$ for prostate cancer [8, 9], 84\% for breast cancer [12], 97.9\% for urinary bladder cancer [18], 94\% for rectal cancer [15], and $98.7 \%$ for colon cancer [16]. In the majority of these studies 50 units was taken as the cut-off value optimally distinguishing cancerous from intact organ.

To our knowledge, it is the first study to investigate TRIMprob for detection of HCC. In our study HCC was responsible for significant lowering of the TRIMprob signal in comparison with healthy liver. It is in accordance with other studies, in which cancers reduced the signal intensity recorded by TRIMprob. It should be noted that we tested TRIMprob for detection of advanced HCC because the mean diameter of the tumour was $4.2 \mathrm{~cm}$. The performance of TRIMprob in detecting early HCC measuring 1-2 cm (target of ultrasound surveillance) with lower load of rapidly dividing cells remains unknown.

In comparison to other cancers HCC shows several peculiarities, which may be important for TRIMprob measurements. First, the liver is a voluminous organ and HCC may be situated deep under the skin surface. The influence of the distance from the probe to the tumour on signal intensity is not well known. In all published studies TRIMprob was used to search for cancers located in close proximity to the probe in such organs as breast, rectum, thyroid gland, or prostate. Second, in almost all cases HCC arises in cirrhotic liver, while cancers in other organs develop in an intact background. This may be a reason for the considerably higher cut-off value discriminating cancer and non-cancer patients in relation to other studies (106 vs. about

Table II. TRIMprob signal intensities according to the wave frequency and compared groups of subjects

\begin{tabular}{|c|c|c|c|c|c|c|c|c|c|c|}
\hline \multirow[t]{2}{*}{$\begin{array}{l}\text { Frequency } \\
\text { wave } \\
{[\mathrm{MHz}]}\end{array}$} & \multicolumn{3}{|c|}{$\begin{array}{c}\text { Signal intensity in investigated } \\
\text { groups [units] } \\
\text { mean } \pm \text { SD (median) }\end{array}$} & \multirow[t]{2}{*}{$\begin{array}{l}\text { Cut-off } \\
\text { value } \\
\text { [units] }\end{array}$} & \multirow[t]{2}{*}{$\begin{array}{c}\text { Sensitivity } \\
\text { (\%) }\end{array}$} & \multirow[t]{2}{*}{$\begin{array}{c}\text { Specificity } \\
\text { (\%) }\end{array}$} & \multirow[t]{2}{*}{ PPV (\%) } & \multirow[t]{2}{*}{ NPV (\%) } & \multirow[t]{2}{*}{ AUROC } & \multirow[t]{2}{*}{$P$-value } \\
\hline & $\begin{array}{l}\text { Cirrhosis } \\
\text { with HCC }\end{array}$ & $\begin{array}{l}\text { Cirrhosis } \\
\text { w/o HCC }\end{array}$ & Controls & & & & & & & \\
\hline \multirow[t]{2}{*}{465} & $67.0 \pm 67.2$ & $113 \pm 59.6$ & $162 \pm 32.6$ & 106 & 80 & 94 & 80 & 94 & 0.865 & $<0.0001^{a}$ \\
\hline & $(32.5)$ & $(128)$ & $(160)$ & 106 & 80 & 66 & 40 & 92 & 0.713 & $<0.05^{b}$ \\
\hline \multirow[t]{2}{*}{1395} & $98.3 \pm 35.6$ & $57.6 \pm 47.7$ & $102 \pm 42.5$ & 120 & 90 & 45 & 33 & 94 & 0.571 & $0.4619^{a}$ \\
\hline & (102) & (33) & (112) & 79 & 90 & 63 & 41 & 96 & 0.731 & $<0.005^{b}$ \\
\hline
\end{tabular}

${ }^{a}$ Comparison between cirrhosis with HCC and controls; ${ }^{b}$ Comparison between cirrhosis with HCC and cirrhosis alone. PPV - positive predictive value, NPV - negative predictive value, AUROC - area under ROC curve, w/o - without. 
50 units). Until now, the influence of cirrhosis itself on TRIMprob recordings has been unclear. In our study the patients with cirrhosis and no evidence of HCC at imaging examinations showed decreased TRIMprob signal intensity, providing intermediate results between HCC and healthy liver. In physiological conditions the liver is an organ of low proliferative activity, but in cirrhosis cell proliferation may be enhanced as a sign of regeneration activity. Therefore, it may be speculated that decreased intensity of resonance signal is result of regenerative activity of cirrhotic liver and possibly its pre-neoplastic propensity. Interestingly, cirrhosis was associated with decreasing TRIMprob signal intensity with higher wave frequencies, and recordings at $1395 \mathrm{MHz}$ correlated with albumin and bilirubin serum levels, the parameters reflecting liver function. These findings suggest that TRIMprob should be tested in the assessment of severity of liver fibrosis.

Evidence that cirrhosis itself is responsible for reduction of TRIMprob decreases the diagnostic ability of this method to find HCC within cirrhotic liver. Nonetheless, TRIMprob showed satisfactory performance in detection of HCC in cirrhotic patients with $80 \%$ sensitivity. Low (66\%) specificity could be entirely attributed to the effect of cirrhosis on resonance interaction. However, the specificity to detect HCC was significantly improved by accepting a two-step approach, using in the same patient with two different wave frequencies $-465 \mathrm{MHz}$ and $1395 \mathrm{MHz}$.

\section{Conclusions}

TRIMprob is a diagnostic technique that may identify HCC within cirrhotic liver; however, the accuracy of this method in screening circumstances may be hindered by attenuation of the resonance signal by cirrhosis itself.

\section{Acknowledgments}

We thank Mr Mateusz Trybulec and Mr Stan Dembowski from Echosens for delivering the TRIMprob device and technical assistance.

\section{Conflict of interest}

The authors declare no conflict of interest.

\section{References}

1. El-Serag HB. Hepatocellular carcinoma. N Engl J Med 2011; 365: 1118-27.

2. Llovet JM, Ducreux M, Lencioni R, et al. European Association for the Study of the Liver and European Organisation for Research and Treatment of Cancer. EASL-EORTC Clinical Practice Guidelines: management of hepatocellular carcinoma. J Hepatol 2012; 56: 908-43.
3. Naghavi M. The global burden of cancer 2013. JAMA Oncol 2015; 1: 505-27.

4. Thun MJ, De Lancey JO, Center MM, et al. The global burden of cancer: priorities for prevention. Carcinogenesis 2010; 31: 100-10.

5. Vedruccio C, Meessen A. EM cancer detection by means of non-linear resonance interaction. Proceedings of the Progress in Electromagnetics Research Symposium (PIERS) 2004; Pisa, Italy, March 28-31: 909-12.

6. Pokorny J, Vedruccio C, Cifra M, et al. Cancer physics: diagnostics based on damped cellular elastoelectrical vibrations in microtubules. Eur Biophys I 2011; 40: 747-59.

7. Bellorofonte C, Vedruccio C, Tombolini P, et al. Non-invasive detection of prostate cancer by electromagnetic interaction. Eur Urol 2005; 47: 29-37.

8. Da Pozzo L, Scattoni V, Mazzoccoli B, et al. Tissue-resonance interaction method for the noninvasive diagnosis of prostate cancer: analysis of a multicentre clinical evaluation. BJU Int 2007; 100: 1055-9.

9. Tubaro A, de Nunzio C, Trucchi A, et al. The electromagnetic detection of prostatic cancer: evaluation of diagnostic accuracy. Urology 2008; 72: 340-4.

10. Gokce O, Sanli O, Salmaslioglu A, et al. Tissue resonance interaction method (TRIMprob) has the potential to be used alongside the recognized tests in the screening protocols for prostate cancer. Int J Urol 2009; 16: 580-3.

11. Di Viccaro D, Perugia G, Cerulli C, et al. The accuracy of tissue resonance interaction method probe (Trimprob) in non-invasive diagnosis of prostatic cancer. Analysis of the results of 782 patient. Urology 2009; 76 (Suppl 15): 1-3.

12. De Cicco C, Mariani L, Vedruccio C, et al. Clinical application of spectral electromagnetic interaction in breast cancer: diagnostic results of a pilot study. Tumor 2006; 92: 207-12.

13. Sacco R, Sammarco G, de Vinci R, et al. Relief of gastric cancer with an electromagnetic interaction system (TRIMprob) in outpatients. Sur Ital 2007; 59: 823-8.

14. Sacco R, Innaro N, Pata F, et al. Preoperative diagnosis of incidental carcinoma in multinodular goitre by means of electromagnetic interactions. Sur Ital 2007; 59: 247-51.

15. Vannelli A, Leo E, Battaglia L, et al. Diagnosis of rectal cancer by electromagnetic interactions: preliminary results. Dis Colon Rectum 2009; 52: 162-6.

16. Dore $M$, Tufano $M$, Pes $G$, et al. Tissue resonance interaction accurately detects colon lesions: a double-blind pilot study. World I Gastroenterol 2015; 21: 7851-9.

17. Gervino G, Autino E, Kolomoets E, et al. Diagnosis of bladder cancer at 465 MHz. Electromag Biol Med 2007; 26: 119-34.

18. Cormio L, Vedruccio C, Leucci G, et al. Noninvasive electromagnetic detection of bladder cancer. Urology 2014; 2014: 802328.

19. Mourad A, Deuffic-Burban S, Ganne-Carrie N, et al. Hepatocellular carcinoma screening in patients with compensated hepatitis $\mathrm{C}$ virus ( $\mathrm{HCV}$ )-related cirrhosis aware of their HCV status improves survival: a modeling approach. Hepatology 2014; 59: 1471-81.

20. Bolondi L. Screening for hepatocellular carcinoma in cirrhosis. J Hepatol 2003; 39: 1076-84.

21. Kim CK, Lim JH, Lee WJ. Detection of hepatocellular carcinomas and dysplastic nodules in cirrhotic liver: accuracy of ul- 
trasonography in transplant patients. J Ultrasound Med 2001; 20: 99-104.

22. Trevisani F, De Notariis S, Rapaccini G, et al. Semiannual and annual surveillance of cirrhotic patients for hepatocellular carcinoma: effects on cancer stage and patient survival (Italian experience). Am J Gastroenterol 2002; 97: 734-44.

23. Trevisani F, D'Intino PE, Morselli-Labate AM, et al. Serum alpha-fetoprotein for diagnosis of hepatocellular carcinoma in patients with chronic liver disease: influence of $\mathrm{HbsAg}$ and anti-HCV status. J Hepatol 2001; 34: 570-5.

24. Di Bisceglie AM, Sterling RK, Chung RT, et al. HALT-C Trial Group. Serum alpha-fetoprotein levels in patients with advanced hepatitis C: results from the HALT-C Trial. J Hepatol 2005; 43: 434-41.

Received: 16.12 .2016

Accepted: 28.03 .2017 